\title{
Expression of ARHGEF11 mRNA in Schizophrenic Thalamus
}

\author{
GENOVEVA DAVIDKOVA, ROBERT E. MCCULLUMSMITH, \\ AND JAMES H. MEADOR-WOODRUFF \\ Mental Health Research Institute and Department of Psychiatry, \\ University of Michigan, Ann Arbor, Michigan 48109, USA
}

KEYWORDS: Rho guanine nucleotide exchange factor 11; schizophrenia; thalamus

Human Rho guanine nucleotide exchange factor 11 (ARHGEF11) is a novel PDZ domain-containing Rho GTPase, which belongs to a family of small GTP-binding proteins that has a role in remodeling of the cytoskeleton and signal transduction processes. ${ }^{1,2}$ The rat homologue of ARHGEF11, GTRAP48, has been shown to interact with the $\mathrm{C}$ terminus of the neuronal excitatory amino acid transporter EAAT4 in vitro, and to enhance glutamate transport in vivo, possibly by stabilizing and/or anchoring EAAT4 to the cell membrane, and thereby making it less likely to be internalized and subsequently degraded. ${ }^{3}$ In view of the participation of ARHGEF11 in glutamate transport, we studied of the expression of ARHGEF11 mRNA in postmortem human thalamus from nonpsychiatric controls and schizophrenic individuals obtained from the Mount Sinai Medical Center Brain Bank.

We used a riboprobe in situ hybridization procedure established in our laboratory. ${ }^{4}$ The published sequence for human ARHGEF11 (NCBI Accession NM_014784) was used to design PCR primers (located at nucleotide positions 1935-1955 for the $5^{\prime}$ primer and 2288-2309 for the $3^{\prime}$ primer) to subclone this portion of the ARHGEF11 into the Zero Blunt TOPO vector (Invitrogen). Antisense and sense ARHGEF11 riboprobes were radiolabeled with $\left[{ }^{35}\right.$ S U UTP. Initially, these antisense and sense ARHGEF11 riboprobes were hybridized to sections from several different regions of the macaque brain (thalamus, cerebellum, prefrontal cortex, striatum) to determine the specificity of the probes. Subsequently, the expression of ARHGEF11 mRNA was evaluated in the human postmortem samples. For each individual, duplicate sections were hybridized to the antisense riboprobe and the slides were apposed to Kodak Biomax film for 1 month. In each thalamic section, six discrete nuclei were identified: anterior (A), ventral anterior (VA), dorsomedial (DM), central medial $(\mathrm{CM})$, pooled ventral tier $(\mathrm{V})$, and reticular $(\mathrm{R})$. The levels of ARHGEF11mRNA

Address for correspondence: Genoveva Davidkova, Mental Health Research Institute and Department of Psychiatry, University of Michigan, 205 Zina Pitcher Place, Ann Arbor, MI 48109. Voice: 734-936-2061; fax: 734-647-4130.

gpuzunov@umich.edu

Ann. N.Y. Acad. Sci. 1003: 375-377 (2003). (C) 2003 New York Academy of Sciences. doi: 10.1196/annals.1300.030 


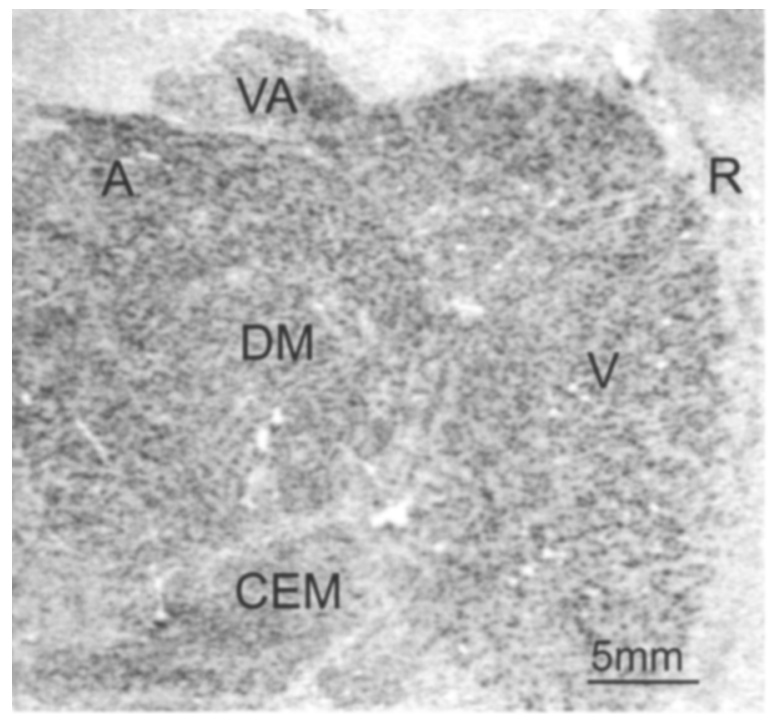

FIGURE 1. ARHGEF11 mRNA expression in human thalamus from a schizophrenic individual. Shown is a photomicrograph of a representative section with the six analyzed thalamic nuclei abbreviated as follows: $\mathrm{A}=$ anterior; $\mathrm{VA}=$ ventral anterior; $\mathrm{V}=$ ventral; $\mathrm{DM}=$ dorsomedial; $\mathrm{CEM}=$ central medial; $\mathrm{R}=$ reticular. Scale bar $=5 \mathrm{~mm}$.

were measured in the individual nuclei and expressed as optical density (OD) units. Statistical analyses were performed using analyses of variance (ANOVA) with nucleus and diagnosis as independent variables and optical density as the dependent variable. For all tests $\alpha=0.05$.

ARHGEF11 mRNA was expressed in a heterogeneous pattern in the human thalamus (FIG. 1). The overall expression levels of ARHGEF11 mRNA were found to be significantly higher in the thalamic nuclei from the schizophrenic subjects as compared to the nonpsychiatric control individuals $\left(\mathrm{F}_{\text {diagnosis }}=11.7, P=.001\right)$ (FIG. 2$)$. Highest levels of ARHGEF11 mRNA were found in the dorsomedial, followed by the anterior nucleus $\left(\mathrm{F}_{\text {nuclei }}=4.1, P=.0021\right)$. The lowest levels of ARHGEF11 mRNA were found in the reticular nucleus in both nonpsychiatric controls and schizophrenic individuals. The expression levels of ARHGEF11 varied to a greater extent among the six examined nuclei in schizophrenic subjects, whereas in the nuclei of the control subjects the expression levels were more homogeneous.

To our knowledge, this is the first in situ hybridization study of the expression of ARHGEF11 in postmortem human thalamus. Since the highest levels of ARHGEF11 mRNA were found in the dorsomedial and in the anterior thalamic nuclei of schizophrenic individuals, and these nuclei have connections to the limbic system, it is reasonable to speculate that ARHGEF11 is involved in the pathologic changes and associated with decreased glutamatergic neurotransmission in schizophrenia. These same subjects were previously found in our laboratory to express altered levels of certain NMDA receptor subunit and postsynaptic density (PSD) protein mRNAs. ${ }^{5} \mathrm{To}$ 


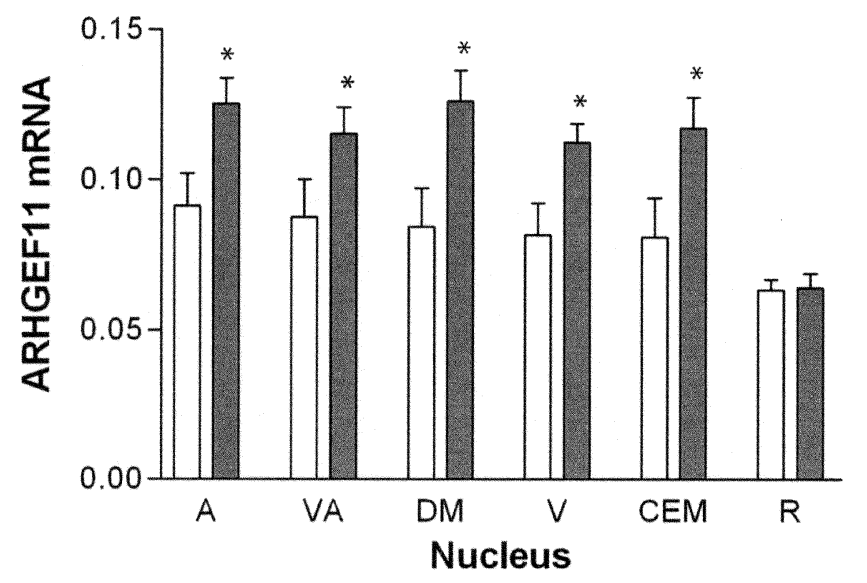

FIGURE 2. ARHGEF11 levels in thalamic nuclei from nonpsychiatric control and schizophrenic individuals. Each bar represents optical density (OD) values expressed as mean \pm SEM from six control and six schizophrenic individuals. The abbreviations of the nuclei have been indicated in the legend to FIgURE 1. Statistical analysis showed that ARHGEF11 levels are significantly higher in the schizophrenic individuals as compared to the nonpsychiatric controls $\left(\mathrm{F}_{\text {diagnosis }}=11.7, P=.001\right)$. Open bars, control; filled bars, schizophrenic. $P=.05$.

evaluate further the significance of ARHGEF11 for the complex molecular glutamatergic changes in schizophrenia, we plan to compare the cellular localization of ARHGEF11 mRNA with the localization of EAAT4 mRNA, as well as examine the expression of similar molecules in these subjects.

\section{REFERENCES}

1. Fukuhara, S. et al. 1999. A novel PDZ domain containing guanine nucleotide exchange factor links heterotrimeric G proteins to Rho. J. Biol. Chem. 274: 58685879.

2. Rumenapp, U. et al. 1999. Rho-specific binding and guanine nucleotide exchange catalysis by KIAA0380, a Dbl family member. FEBS Lett. 459: 313-318.

3. JACKSON, M. et al. 2001. Modulation of the neuronal glutamate transporter EAAT4 by two interacting proteins. Nature 410: 89-93.

4. Meador-Woodruff, J.H et al. 1996. Differential regulation of hippocampal AMPA and kainite receptor subunit expression by haloperidol and clozapine. Mol. Psychiatry 1: 41-53.

5. Meador-Woodruff, J.H. \& J.E. Kleinman. 2002. Neurochemistry of schizophrenia: glutamatergic abnormalities. In Neuropsychopharmacology: The Fifth Generation of Progress. K.L. Davis, et al., Eds.: 717-728. American College of Neuropsychopharmacology. 